\title{
A molecular dynamics study on the tribological behavior of molybdenum disulfide with grain boundary defects during scratching processes
}

\author{
Boyu WEI ${ }^{1}$, Ning KONG ${ }^{1, *}$, Jie ZHANG ${ }^{1}$, Hongbo LI ${ }^{1}$, Zhenjun HONG ${ }^{1}$, Hongtao ZHU $^{2}$, Yuan ZHUANG \\ Bo WANG ${ }^{3}$ \\ ${ }^{1}$ School of Mechanical Engineering, University of Science and Technology Beijing, Beijing 100083, China \\ ${ }^{2}$ School of Mechanical, Materials \& Mechatronics Engineering, University of Wollongong, Wollongong, NSW 2522, Australia \\ ${ }^{3}$ Beijing Institute of Spacecraft System Engineering, Beijing 100094, China
}

Received: 14 June 2020 / Revised: 26 August 2020 / Accepted: 28 September 2020

(C) The author(s) 2020 .

\begin{abstract}
The effect of grain boundary (GB) defects on the tribological properties of $\mathrm{MoS}_{2}$ has been investigated by molecular dynamics (MD) simulations. The GB defects-containing MoS2 during scratching process shows a lower critical breaking load than that of indentation process, owing to the combined effect of pushing and interlocking actions between the tip and $\mathrm{MoS}_{2}$ atoms. The wear resistance of $\mathrm{MoS}_{2}$ with GB defects is relevant to the misorientation angle due to the accumulation of long Mo-S bonds around the GBs. Weakening the adhesion strength between the $\mathrm{MoS}_{2}$ and substrate is an efficient way to improve the wear resistance of $\mathrm{MoS}_{2}$ with low-angle GBs.
\end{abstract}

Keywords: $\mathrm{MoS}_{2}$; grain boundary; tribological behavior; molecular dynamics; misorientation angle

\section{Introduction}

In recent years, two-dimensional (2D) transition metal dichalcogenides (TMDCs) have caused great attentions due to their excellent physical, mechanical and tribology properties [1-5]. As a representative of TMDCs, $\mathrm{MoS}_{2}$ has a layered hexagonal crystal structure, which consists of one metal Mo layer and two S layers [6]. Due to its strong covalent bonding in molecular layer [7], single-layer $\mathrm{MoS}_{2}\left(\mathrm{SL}-\mathrm{MoS}_{2}\right.$ ) shows extraordinary mechanical properties, such as the high effective in-plane Young's modulus $[6,8]$ and low bending modulus [9]. The weak van der Waals interactions in the interlayer of $\mathrm{MoS}_{2}$ make shear more easily, which is the main reason for the low friction of $\mathrm{MoS}_{2}$ [10-12]. Base on the excellent mechanical and tribological properties, $\mathrm{MoS}_{2}$ is commonly used as solid lubricant [13] and wear resistant coating [14], and is utilized as an additive in lubricating oils [13]. Due to the monolayer thickness, SL-MoS 2 and thin film $\mathrm{MoS}_{2}$ present a number of potential applications to serve as lubricant materials in microelectromechanical systems (MEMS) [10, 11]. Under specific conditions, SL-MoS 2 and thin film $\mathrm{MoS}_{2}$ even show a lower friction than graphene [15, 16]. Moreover, the super-lubricity phenomena are observed in $\mathrm{MoS}_{2}-\mathrm{MoS}_{2}$ [17] and $\mathrm{MoS}_{2}-\mathrm{Sb}$ [18] contacts as well.

$\mathrm{SL}_{-\mathrm{MoS}_{2}}$ with high-quality and large-area is required to achieve the application potentials above. However, it is still an enormous challenge to synthesize pristine (without GB defects) SL-MoS2 with large-area by most advanced technologies, including chemical vapor deposition (CVD) and chemical exfoliation $[19,20]$. Chemical exfoliation

* Corresponding author: Ning KONG, E-mail: kongning@ustb.edu.cn 
is used for the small flakes of MoS2. Inevitably, it produces $\mathrm{MoS}_{2}$ with native crystalline defects [21, 22]. The CVD synthesis is able to product large-area $\mathrm{SL}_{-} \mathrm{MoS}_{2}$, but bicrystals and polycrystalline microstructures exist in the $\mathrm{SL}-\mathrm{MoS}_{2}$ due to the multiple nucleation sites on substrates [23, 24]. When adjacent $\mathrm{MoS}_{2}$ crystals merge during the growth process, the grain boundaries (GBs) can be formed [20, 25, 26]. The GB defects, as well as point defects, dislocations and edges are classified as the intrinsic structural defects in SL-MoS2 [27, 28]. The tribological properties of $\mathrm{MoS}_{2}$ are sensitive to the testing and environment conditions, such as the pre-strain within $\mathrm{MoS}_{2}$ [29-31], the magnitude and direction of friction velocity [32-34], the number of $\mathrm{MoS}_{2}$ layers [32] and temperatures [35]. The intrinsic structural defects of $\mathrm{MoS}_{2}$ have been deemed to be detrimental to its strength [24, 36-38]. Therefore, it is meaningful to investigate the effects of GB defects on the tribological behavior of $\mathrm{MoS}_{2}$ for its potential tribology applications. It has been observed with AFM that the friction at the GB is obviously higher than that of pristine SL-MoS2, possibly due to the defects at the GB [39]. In addition, the GBs of polycrystalline $\mathrm{MoS}_{2}$ could cause a layerdependent oscillatory friction, on account of the presence and absence of GBs in odd and even numbers of layers [40]. However, the wear mechanisms of the GB structure are not entirely understood. The study on the wear mechanisms of SL-MoS 2 with GB defects is urgently needed to provide guidelines for enhancing its tribological performances.

In this work, the effect of the GB defects on the tribological behavior of $\mathrm{MoS}_{2}$ has been investigated by means of MD simulations. According to previous theoretical and experimental findings, eight specified zigzag-oriented GBs have been generated in different misorientation angles [20, 21, 37, 41]. The atomistic indentation and scratching processes have been simulated in order to calculate the carrying capacity and wear resistance of $\mathrm{MoS}_{2}$ with different GB types. The results show that GB defects-containing $\mathrm{MoS}_{2}$ is more easily broken during the scratching process owing to the combined effect of pushing and interlocking actions between the diamond tip and $\mathrm{MoS}_{2}$ atoms. $\mathrm{MoS}_{2}$ with a low misorientation angle GB shows a weaker wear resistance due to the accumulation of the long Mo-S bonds around the GBs. Weakening the adhesion strength between $\mathrm{MoS}_{2}$ and substrate is an efficient way to mitigate the negative effects of GBs on the wear resistance of $\mathrm{MoS}_{2}$.

\section{Simulation methods}

In the atomistic models, a diamond tip is scratching against the SL-MoS2 with GB defects. The SL-MoS 2 is coated on a deformable metallic $\mathrm{Pt}$ substrate, as shown in Fig. 1(a). The tip is cut from a diamond crystal with a (111) orientation, and its diameter is $30 \AA$. The dimensions of the SL-MoS 2 and the Pt (111) substrate are approximately $194.0 \AA \times$ $148.0 \AA$ in the $x$ and $y$ directions, and $199.8 \AA \times$ $153.8 \AA \times 72.5 \AA$, respectively. The Pt (111) substrate contains 152,064 platinum atoms. For the SL-MoS 2 (including the $\mathrm{MoS}_{2}$ with and without GB defects), the total number of $\mathrm{Mo}$ and $\mathrm{S}$ atoms ranges from 9,896 to 10,395 . The SL-MoS 2 was placed above the substrate with an initial distance of $2.8 \AA$ where their interaction energy was minimal. During the relaxation process, the diamond tip was placed $15 \AA$ above the $\mathrm{MoS}_{2}$ layer to insure there was no interaction between the tip and SL-MoS2 layer. The $\mathrm{Pt}$ atoms which were on the bottom layer were constrained on the substrate to support the model, as shown in Fig. 1(a). The Mo and S atoms at the edge of the SL-MoS 2 were fixed in the $x$ and $y$ directions, while they can move freely in the $z$ direction to prevent translational movement of the SL-MoS2, as shown in Fig. 1(c). Periodic boundary condition was used along the $x$ and $y$ directions. The Langevin thermostat was applied to the atoms (circled in light-blue in Fig. 1) which were away from the contact region [42, 43]. This thermostat scheme in this work did not show artificial effects compared with another alternative scheme, as shown in Fig. S1 (in the Electronic Supplementary Material, ESM). It should be noted that the Langevin thermostat was used in an NVE ensemble, so as to 

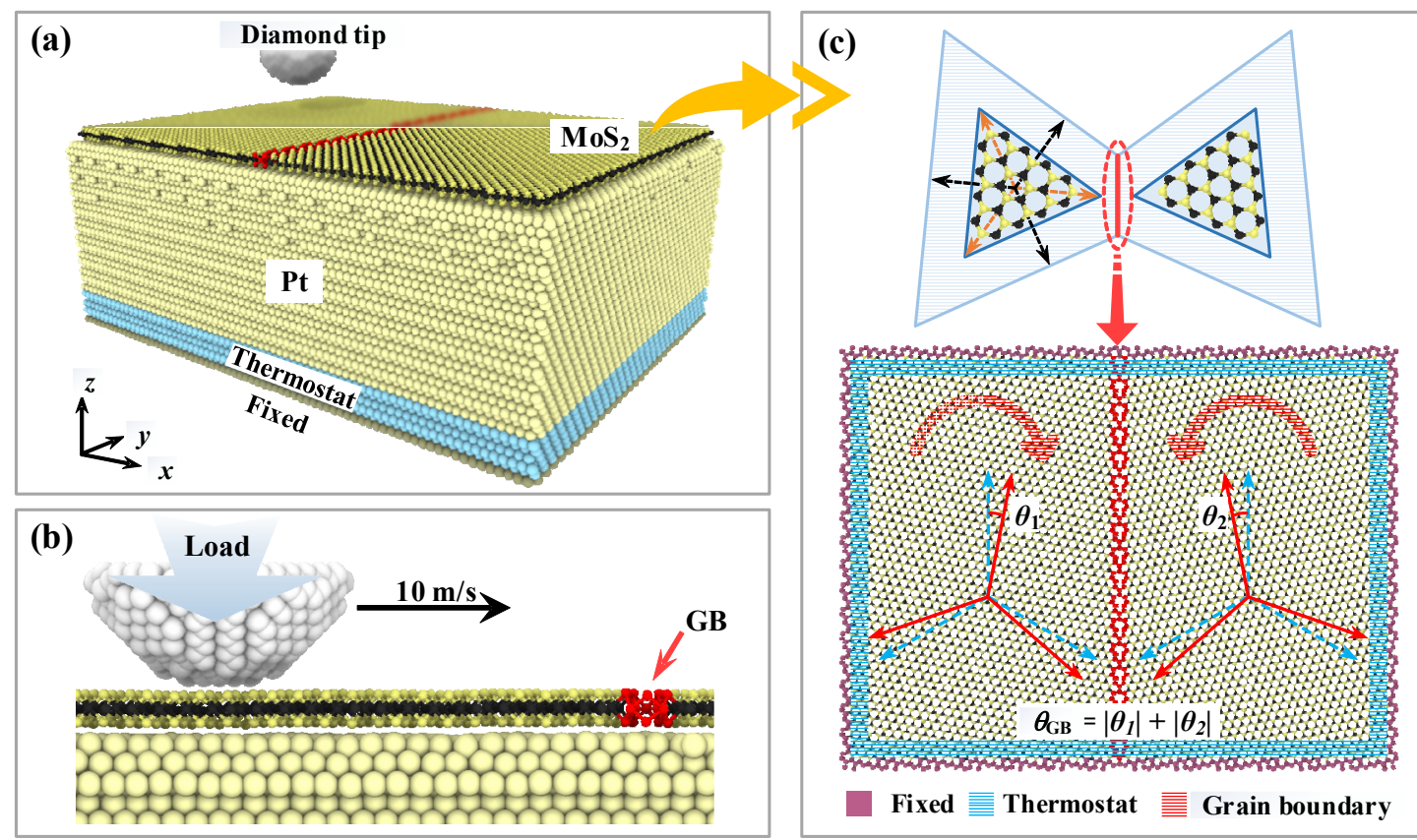

Fig. 1 (a, b) Simulation model of a diamond tip scratching against the GB defects-containing SL-MoS 2 that is coated on the Pt substrate, and (c) schematic of GB's formation process in bicrystal $\mathrm{MoS}_{2}$.

maintain a constant temperature of $300 \mathrm{~K}$.

During the process of $\mathrm{MoS}_{2}$ crystal growth, GBs are formed when adjacent $\mathrm{MoS}_{2}$ crystals merge due to the different orientation angles and sizes of $\mathrm{MoS}_{2}$ crystals grains [25, 26]. Figure 1(c) shows a schematic of GB's formation process in bicrystal $\mathrm{MoS}_{2}$. The GBs of $\mathrm{MoS}_{2}$ crystals are composed of diverse dislocation cores which are dependent on the boundary misorientation angles of crystals. The dislocation cores not only include the conventional pentagon-heptagon (5|7) pairs [21, $41]$, but also exist in the form of quadranglequadrangle (4|4), quadrangle-hexagon (4|6), quadrangleoctagon (4|8) and hexagon-octagon (6|8) pairs [27, $28,37,44]$. In contrast to graphene with 6-fold symmetry, $\mathrm{MoS}_{2}$ with 3-fold symmetry has two types of $5 \mid 7$ dislocation $[37,45]$. In a microscopical view, there are two types of dislocation cores of the $5 \mid 7$ pairs, including the Mo-rich 5I7 type (Mo5|7) and S-rich 5।7 type (S5|7) [37], as shown in Figs. 2(c, d). The zigzag-oriented SL-MoS 2 shows better tribological properties than other orientation [32-34]. In order to deeply study the GB defects' effect on the tribological performances of $\mathrm{MoS}_{2}$, the GB defects are formed in the zigzag-oriented bicrystal $\mathrm{MoS}_{2}$. In terms of the coincidence site lattice (CSL) theory [46], eight specified GBs (consist of Mo5I7 and S5I7 pairs, respectively) were generated in various misorientation angles, as shown in Figs. 2(a, b). For the specified misorientation angle of two adjacent $\mathrm{MoS}_{2}$ crystals, some periodic coincident points along a line could be found. An initial GB can be obtained by cutting the two $\mathrm{MoS}_{2}$ crystals along the line [46]. In order to further optimize the GB's structures, the potential energy of the bicrystal $\mathrm{MoS}_{2}$ has been minimized in the way of the conjugate gradient method. For convenience of representation, the zigzag-oriented GBs which consist of Mo5।7 cores are defined as M-51, M-94, $\mathrm{M}-132$, and $\mathrm{M}-218$, the misorientation angles $\left(\theta_{\mathrm{GB}}\right)$ of which are $5.1^{\circ}, 9.4^{\circ}, 13.2^{\circ}$, and $21.8^{\circ}$, respectively, as shown in Fig. 2(a). Correspondingly, the reversed GBs which consist of S5I7 cores are defined as S-51, S-94, S-132, and S-218 corresponding to $\theta_{\mathrm{GB}}=5.1^{\circ}$, $9.4^{\circ}, 13.2^{\circ}$, and $21.8^{\circ}$, as shown in Fig. $2(\mathrm{~b})$. The schematic of the reversed GB could be found in Fig. S2 in ESM [44].

The intra-layer interactions within $\mathrm{MoS}_{2}$ were described by the REBO potential $[47,48]$, which has 


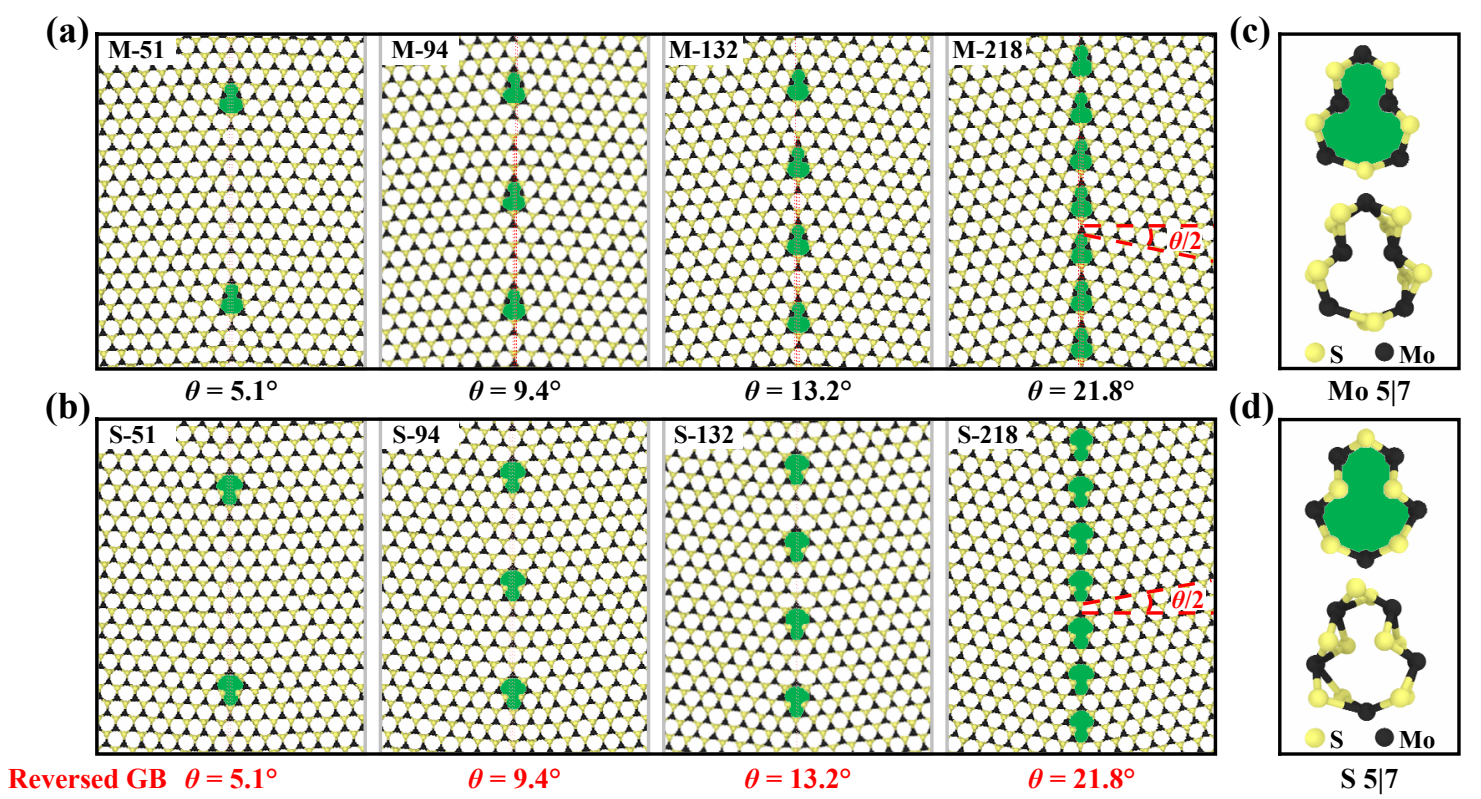

Fig. 2 (a, b) Atomic structures of GB defects in bicrystal $\mathrm{MoS}_{2}$ with different misorientation angles and (c, d) typical dislocation cores (Mo5|7 and S5|7 pairs) captured from GB in bicrystal $\mathrm{MoS}_{2}$, and they are named in terms of the ring pairs characteristics, respectively.

been developed to simulate the break and recombination behaviors of Mo-S bonds and describe MoSz's covalently bonded systems [49]. The EAM potential for $\mathrm{Pt}$ system was applied to evaluate the interactions within the $\mathrm{Pt}$ substrate. The LJ potentials were used to model the interactions among the tip, SL-MoS 2 and the substrate. For C-Mo and C-S interactions, the LJ parameters of $\sigma_{\mathrm{C}-\mathrm{Mo}}=3.01 \AA, \varepsilon_{\mathrm{C}-\mathrm{Mo}}=48.86 \mathrm{meV}$, $\sigma_{\mathrm{C}-\mathrm{S}}=3.42 \AA$, and $\varepsilon_{\mathrm{C}-\mathrm{S}}=13.12 \mathrm{meV}$ were used, respectively [30, 50]. For $\mathrm{Mo}-\mathrm{Pt}, \mathrm{S}-\mathrm{Pt}$, and $\mathrm{C}-\mathrm{Pt}$ interactions, the LJ parameters could be found in Table S1 in ESM [30]. Before indentation process, the whole system was well relaxed for 40 ps at $300 \mathrm{~K}$. For the indentation course, the diamond tip was placed right above the GB of bicrystal $\mathrm{MoS}_{2}$ and moved vertically to the GBs. For the scratching process, the diamond tip was positioned at an initial distance of $50 \AA$ from the GB along the $x$ direction to avoid the tip affecting the GB during the loading process, and moved laterally at different scratching depths. The total scratching distance of the diamond tip was $80 \AA$ to ensure the diamond tip slides across the GB of bicrystal $\mathrm{MoS}_{2}$ completely. The scratching depths were controlled by the tip's vertical displacement.
The speed of the diamond tip was set to $10 \mathrm{~m} / \mathrm{s}$ in the process of indentation and scratch. Simulations have been performed based on the molecular dynamics simulation package LAMMPS. Every simulation has been repeated at least three times along different indentation positions and scratching routes to ensure the reliability of the results, which could be found in Fig. S3 in ESM. The tip atoms usually fall into different contact geometries with different GBs, the critical normal loads show small variations when the tip indents at different positions and scratches along different routes, as shown in Table S2 in ESM. It should be noted that the following results are based on indentation position 1 and scratching route 1 without further mention.

\section{Results and discussion}

\subsection{The indentation and scratching process of $\mathrm{MoS}_{2}$ with GBs}

In order to research the GB defects' effects on the mechanical and tribological properties of $\mathrm{MoS}_{2}$, the atomistic nano-indentation and nano-scratching 
process of the bicrystal $\mathrm{MoS}_{2}$ with GBs have been investigated. Figure 3(a) shows the schematic of indentation process. The diamond tip was placed right above the GB before indenting. In contrast, the tip was initially positioned away from the GB during scratching process, as shown in Fig. 3(b). According to previous tribological MD simulations on $2 \mathrm{D}$ materials, the bond permanent break has been used to characterize the indentation and scratching failure of 2D materials [30, 51-53]. In this work, the critical breaking loads of indentation and scratch were identified by the permanent break of Mo-S bonds, as shown in Fig. 3(c). For the pristine zigzag-oriented SL-MoS2, the breaking loads of indentation and scratching process are respectively 106.6 and $94.4 \mathrm{nN}$, which are close to the previous works $[30,50]$. Compared with the pristine $\mathrm{MoS}_{2}$, the critical breaking loads for indentation on M-51 and S-51 models decrease by $19.37 \%$ and $27.12 \%$, respectively. During the
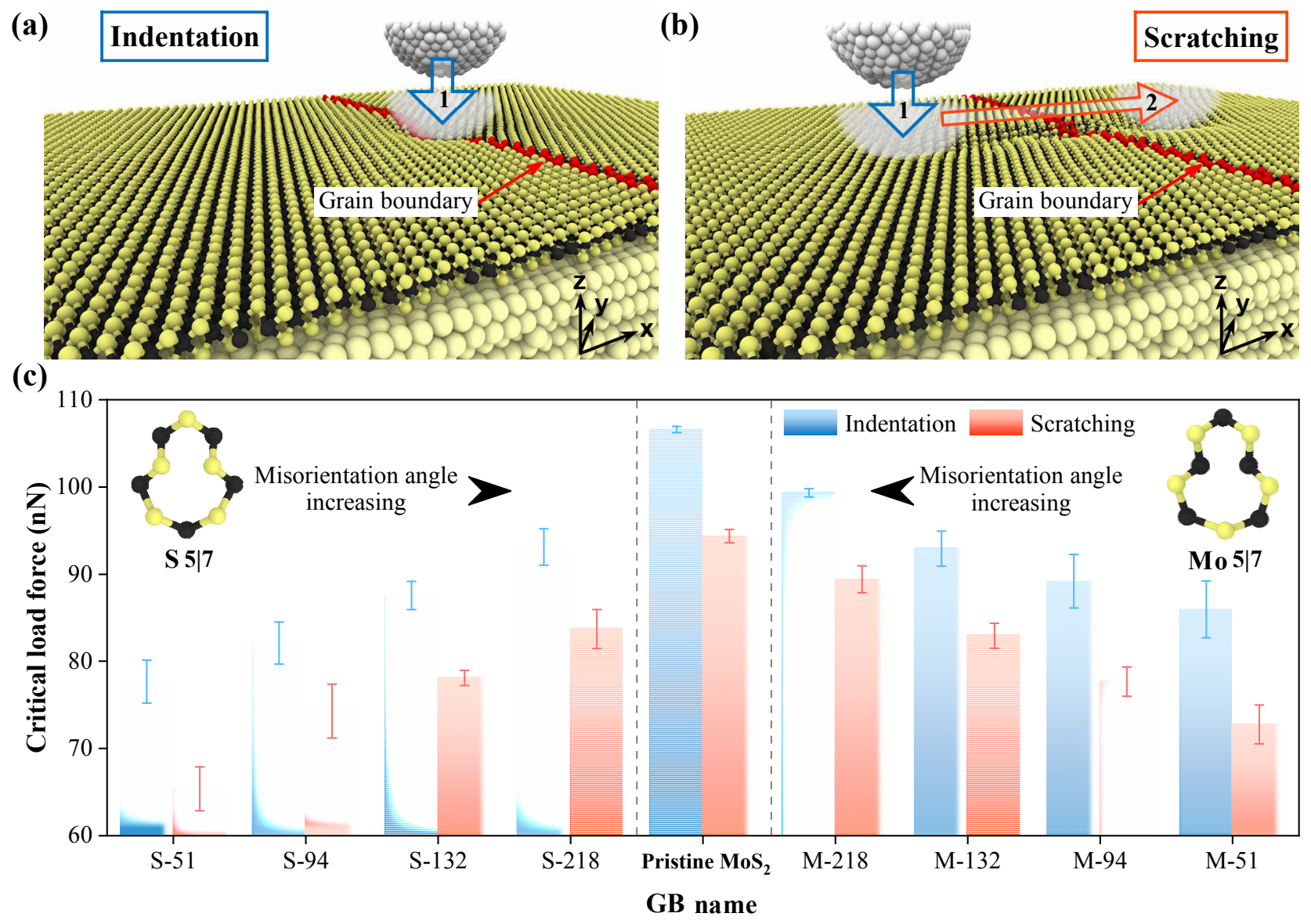

Fig. 3 Schematic diagrams of (a) indenting and (b) scratching processes. (c) Critical breaking loads of pristine MoS 2 and $\mathrm{GB}$ defects-containing $\mathrm{MoS}_{2}$. The values were obtained by averaging the results from the repeated simulations.

indentation process, the critical breaking loads of GB defects-containing SL-MoS 2 increase with the misorientation angle. When the misorientation angle is greater than $20^{\circ}$, the critical breaking loads for indentation and scratching are close to those of pristine $\mathrm{MoS}_{2}$. More interestingly, the critical breaking loads of all GB defects-containing $\mathrm{MoS}_{2}$ during the scratching process are lower than those during indentation. It indicates scratching action imposes more extreme working condition than indentation for $\mathrm{MoS}_{2}$.

To understand the reason of the lower critical breaking load for GB defects-containing $\mathrm{MoS}_{2}$ during scratching process, $\mathrm{MoS}_{2}$ structural deformations for indentation and scratching have been investigated. Unlike graphene has only one C atom layer, SL-MoS2 consists of a Mo atom layer sandwiched by two $S$ layers. Therefore, the deformation mechanism of $\mathrm{SL}_{-} \mathrm{MoS}_{2}$ is more complicated, such as the existence of the out-of-plane 
compression deformation of $\mathrm{SL}_{-\mathrm{MoS}_{2}}$ [54]. The structural deformations of $\mathrm{MoS}_{2}$ could be better described with the length of Mo-S bond ( $\left.L_{\mathrm{Mo}-\mathrm{S}}\right)$. The out-of-plane compressive and in-plane tensile deformation of $\mathrm{MoS}_{2}$ could be described by the $L_{\mathrm{S}-\mathrm{S}}$ (distance between the relative $\mathrm{S}$ atoms) and $L_{\mathrm{Mo-Mo}}$ (distance between the adjacent Mo atoms) [30]. The radial distributions of the $L_{\mathrm{Mo}-\mathrm{S}}, L_{\mathrm{Mo}-\mathrm{Mo}}$, and $L_{\mathrm{S}-\mathrm{S}}$ have been calculated under the same load, which is the critical breaking load of M-51 model during the scratching process (scratch depth of $5.4 \AA$ ), as shown in Figs. $4(\mathrm{a}-\mathrm{c})$. For the indentation course, the structural deformations of $\mathrm{MoS}_{2}$ exhibit a better symmetry, while the deformations of $\mathrm{MoS}_{2}$ display an obvious asymmetry during the scratching process, as shown in Figs. 4(a-c). Severe out-of-plane compressive and in-plane tensile deformations are mostly distributed in front of the diamond tip during scratching process. It should be noted that the scratching process exhibits a larger in-plane tensile deformation than indentation process, as shown in Fig. 4(b). To better represent the deformation, the $\mathrm{MoS}_{2}$ atoms are colored on the basis of the relative displacements in the $x$ direction, as shown in Figs. 4(d) and 4(e). In the contact area [55], $\mathrm{MoS}_{2}$ is stretched under indentation owing to the differences of the atom relative displacement, which is accordance with a previous study [7]. For the scratching process, the $\mathrm{MoS}_{2}$ deforms plastically in the contact area at the same load of indentation. Under the pushing force of diamond tip atoms, the $\mathrm{MoS}_{2}$ atoms in the contact area move forwards along the $x$ direction, which is contrary to the indentation process. A larger local stretching occurs in front of the tip due to the nonuniform relative displacement of $\mathrm{MoS}_{2}$ atoms in the contact area.

The combined effect of pushing and interlocking actions during the scratching process also leads to the difference in the critical breaking load between indentation and scratching processes. Figure 5 shows the failure mechanisms during the scratching process which are caused by pushing and interlocking actions. During the course of scratch, the diamond tip atoms are easily interlocked in the $\mathrm{MoS}_{2}$ lattice due to the abundant hollow positions

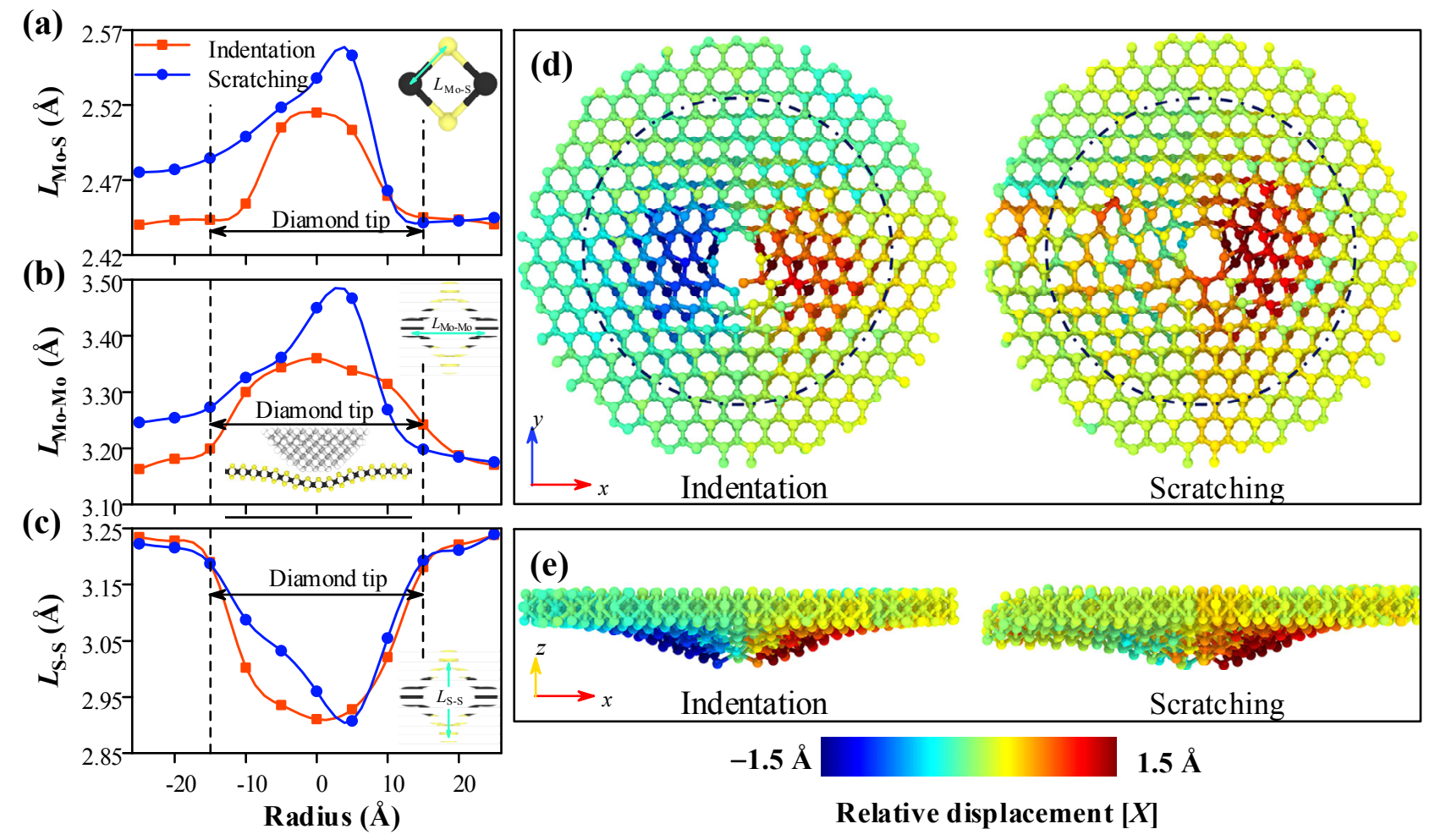

Fig. 4 Radial distributions of (a) $L_{\mathrm{Mo}-\mathrm{S}}$, (b) $L_{\mathrm{Mo}-\mathrm{Mo}}$, and (c) $L_{\mathrm{S}-\mathrm{S}}$ for $\mathrm{MoS}_{2}$ at the load depth of $5.4 \AA$. (d, e) Relative displacements of $\mathrm{MoS}_{2}$ atoms in $x$ direction regarding to their initial positions for indentation and scratching processes of M-51 Model at the load depth of $5.4 \AA$. The edge of the tip was marked by dashed circles. 
in $\mathrm{MoS}_{2}$. The atomistic interlock results in a high friction [42]. When the scratching time $(t)$ is $445 \mathrm{ps,}$ the atom $\mathrm{A}$ of the tip is interlocked with the atom 1 and 2 of MoS2. When $t=470 \mathrm{ps}$, the atom 2 of $\mathrm{MoS}_{2}$ is pushed away by the atom A. Meanwhile, the atom $\mathrm{B}$ is interlocked with the Mo-S bond between the atom 3 and 4 . Then the Mo-S bond is broken because the atom B moves forwards. Similarly, when $t=505 \mathrm{ps}$, the atom $\mathrm{C}$ is interlocked with the atom 5 and 6 . The atom $C$ exerts a lateral force to atom 6 , which leads to a bond break between atom 5 and 6 , as well as breaks the bond nearby which is indicated by a black dotted circle in Fig. 5. Those interlocking and pushing behaviors iteratively occur during the scratching process, which result in a possibility to break the Mo-S bonds of MoS2. In contrast, there are less interlocking and pushing actions because of lacking the interfacial sliding movements during indentation process. Similar interlocking and pushing actions also happen in S-51 model as shown in Fig. S4 in ESM. In addition, the effect of misorientation angles on these above interactions has been further discussed, as shown in Fig. S5 in ESM. For the SL-MoS2 with the low-angle GB, the GB structure shows a greater deformation after the diamond tip slides over the GB. With the misorientation angle increasing, the deformation degree and the number of broken Mo-S bond reduce due to the increase of the GB dislocation density [56].

\subsection{Wear mechanism of $\mathrm{MoS}_{2}$ with GB defects}

Researches show that the pristine SL-MoS2 which is supported on a substrate can effectively reduce the friction $[30,50]$. To investigate the tribological behavior and wear mechanism of $\mathrm{MoS}_{2}$ with GB defects, the diamond tip has been used to scratch each GB defects-containing SL-MoS2. The variations of friction with scratching distance were calculated to show the effect of GB defects on friction, which is shown in Fig. 6(a). For the pristine $\mathrm{MoS}_{2}$, the friction force increases with scratching distance on the early stage of scratching process, and then stays stable. The similar phenomena also can be observed before the tip reaches to GB defects in the GB defects-containing models. Taking M-51

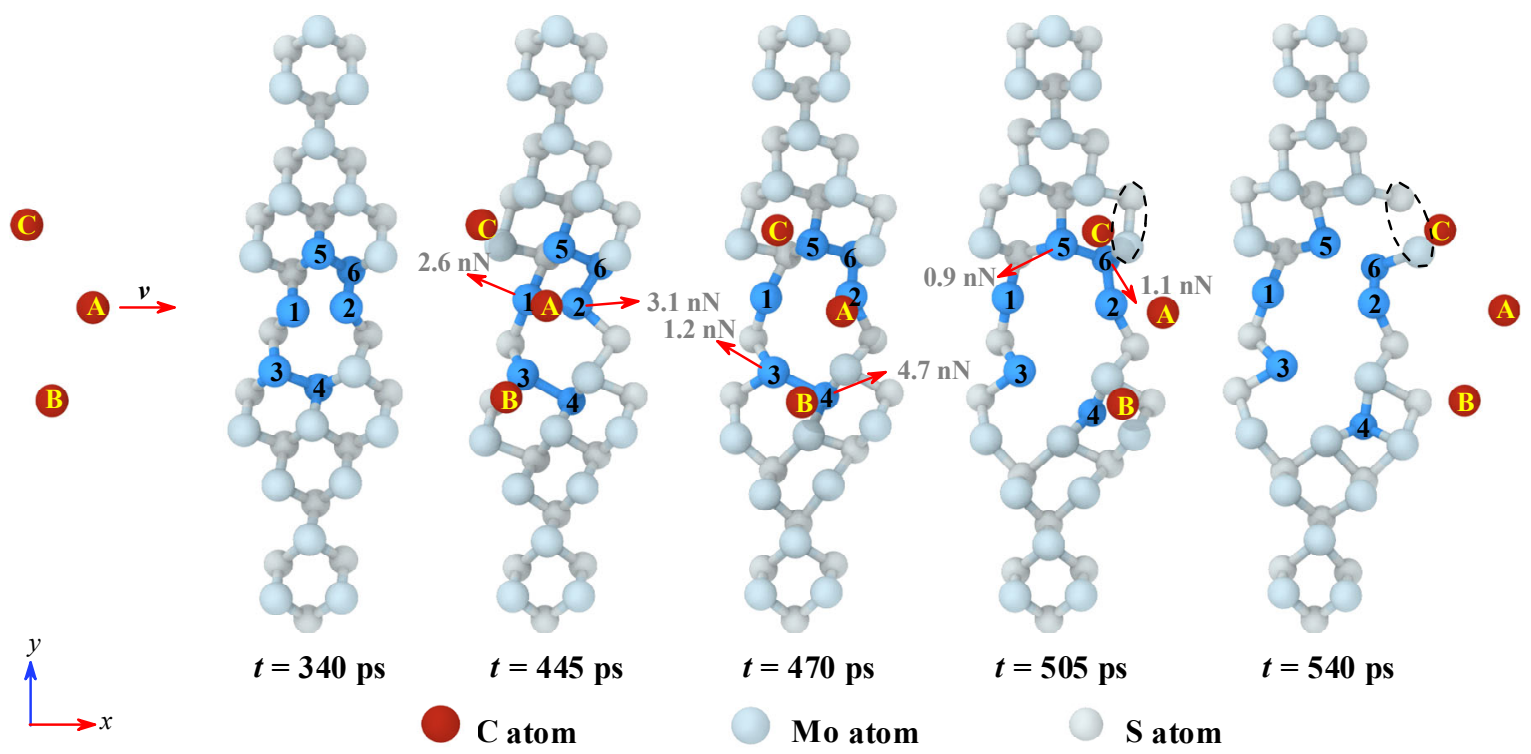

Fig. 5 Schematic of the specific tip atoms and $\mathrm{MoS}_{2}$ atoms of M-51 Model during the scratching process at the load depth of $5.4 \AA$. The top $\mathrm{S}$ layer is hidden to show the structural changes of $\mathrm{MoS}_{2}$ clearly. The representative atoms of the tip and $\mathrm{MoS}_{2}$ were marked by red and blue color, respectively. In order to trace the bond-breaking actions, the interaction force and broken bond were marked by red arrow and black circle, respectively. The interaction force was individually obtained by calculating the interaction between each specific tip atom and specified $\mathrm{MoS}_{2}$ atoms. For display the interaction in a two-dimensional plane, only $x$ and $y$ components were used to find out the resultant force. The detailed interaction forces between the tip atoms and $\mathrm{MoS}_{2}$ atoms could be found in Tables S3 and S4 in ESM. 

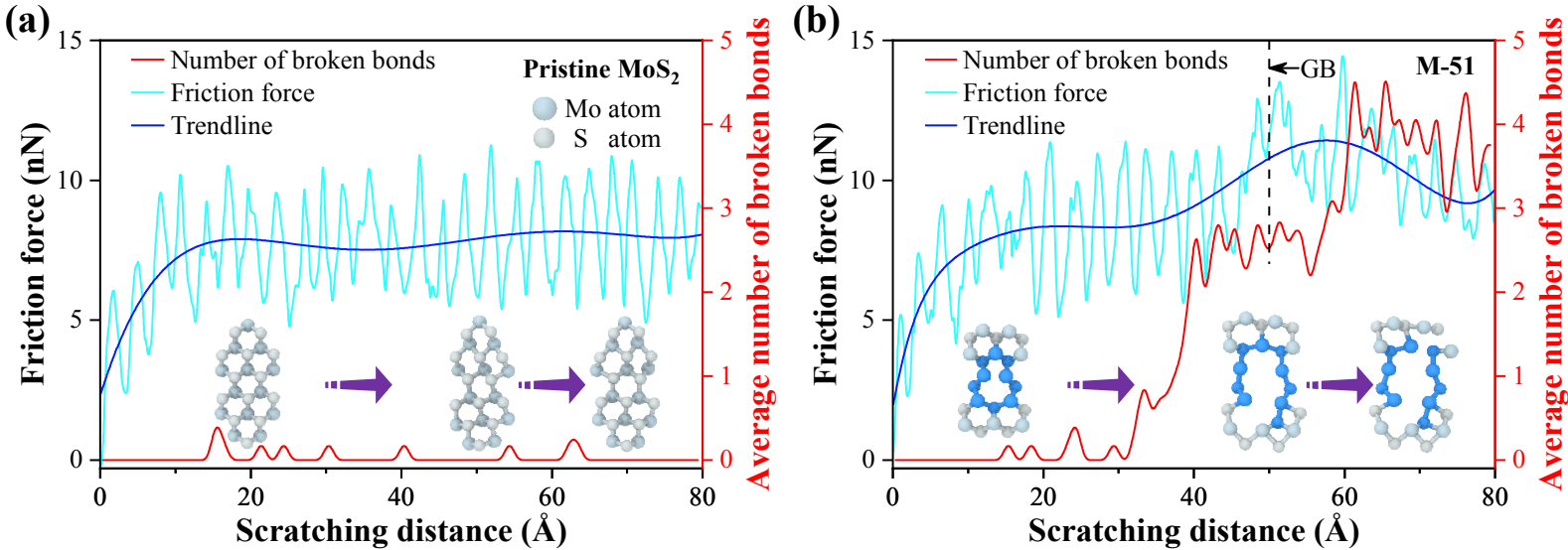

Fig. 6 At a scratching depth of $5.4 \AA$, the change of friction force and the average number of broken Mo-S bonds during the scratching process of (a) pristine $\mathrm{MoS}_{2}$ and (b) M-51 model. The average number of broken bonds is averaged with an interval of $1 \AA$. The top $\mathrm{S}$ layer is hidden to show the structural changes of $\mathrm{MoS}_{2}$ directly. The Mo and S atoms around the Mo5|7 pairs are marked by blue color.

model as an example, the friction force increases and becomes unstable when the tip is close to the GB defects, as shown in Fig. 6(b). The coefficient of friction follows the same trend as the friction force as shown in Fig. S6(a) in ESM. Owing to the lamellar structure of 2D materials the wear is hard to be quantified by the continual volume of material removed. Instead, the number of broken bonds can be used to analyze the wear for 2D materials [53]. Figure 6(b) shows the relationship between the average number of broken Mo-S bonds and scratching distance in M-51 model. The number of broken bonds shows a sharp increase when the tip scratches to GB defects, while it is almost zero in pristine $\mathrm{MoS}_{2}$ model. Therefore, the GB defects could accelerate the local Mo-S bond breaking and rupture initiation of $\mathrm{MoS}_{2}$, which leads to a reduction on the wear resistance of $\mathrm{MoS}_{2}$.

The average friction forces around GB defects were calculated to accurately evaluate the GB defects effect on the anti-friction performance of $\mathrm{MoS}_{2}$. Figure 7(a) shows the relationship between the average friction force and the scratching depth during the scratching process. The scratching processes on the substrate covered with the SL-MoS 2 show an extremely low friction force at a scratching depth below $2 \AA$, which is accordance with the previous study [50]. The average friction force on the GB defects-containing $\mathrm{SL}-\mathrm{MoS}_{2}$ is lower than that on the Pt substrate only, when the scratching depth is below $5 \AA$. It indicates that the $\mathrm{MoS}_{2}$ with GB defects still can be used as an effective solid lubricant at low load. With the increasing scratching depth, a sudden increase of the average friction force happens in all the lines in the Fig. 7(a). It corresponds to a number of Mo-S bond breaks, and then leads to a total failure of $\mathrm{MoS}_{2}$. The critical breaking loads for wear failure are identified by the permanent break of Mo-S bonds. Compared with the pristine $\mathrm{MoS}_{2}$, the critical breaking loads for wear failure on M-51 and S-51 GBs are reduced by $22.9 \%$ and $30.7 \%$, respectively, as shown in Table 1 . The wear resistance of GB defects-containing SL-MoS 2 increases with the misorientation angle, but less than that of the pristine $\mathrm{MoS}_{2}$. The GB dislocation density of bycrystal $\mathrm{MoS}_{2}$ increases with the misorientation angle. The strain fields which are caused by these dislocations can be canceled and overlapped, resulting in the improvement of the GB structural stability [56]. In addition, the failure of $\mathrm{MoS}_{2}$ with GB defects under uniaxial tensile owes to the higher pre-strain in the Mo-S bonds around GBs [37]. The longest Mo-S bond length of each model has been calculated as shown in Fig. S7 in ESM. The longest bond length decreases with the increase of the misorientation angle. However, the $\mathrm{MoS}_{2}$ layers under the diamond tip suffers more complex local strains and stresses 
(a)

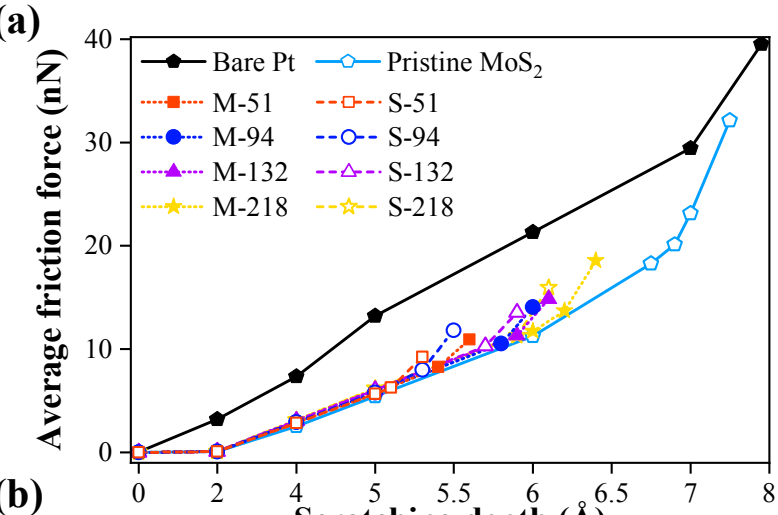

(b)

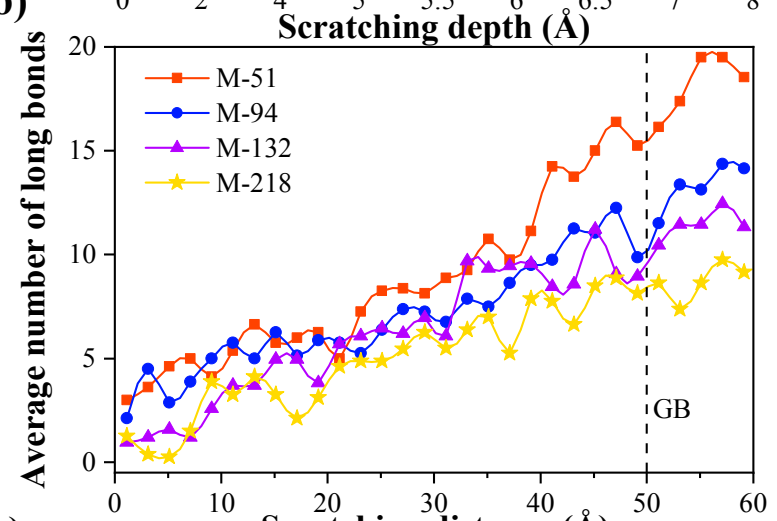

(c)

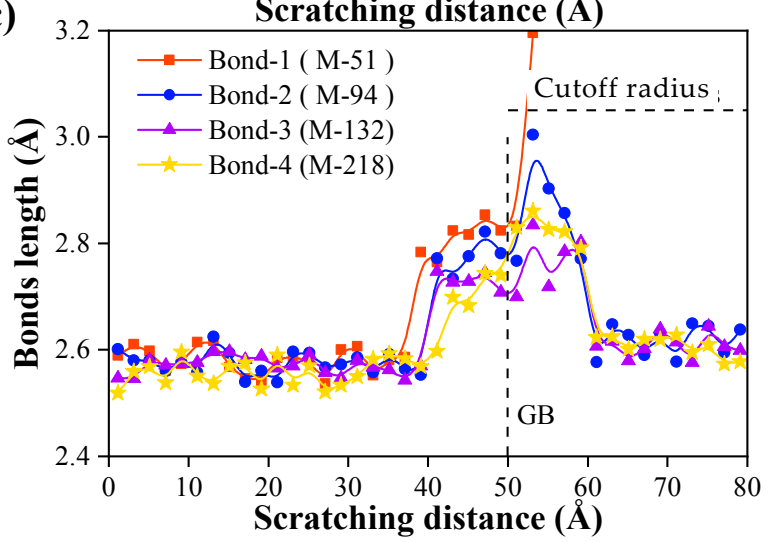

(d)

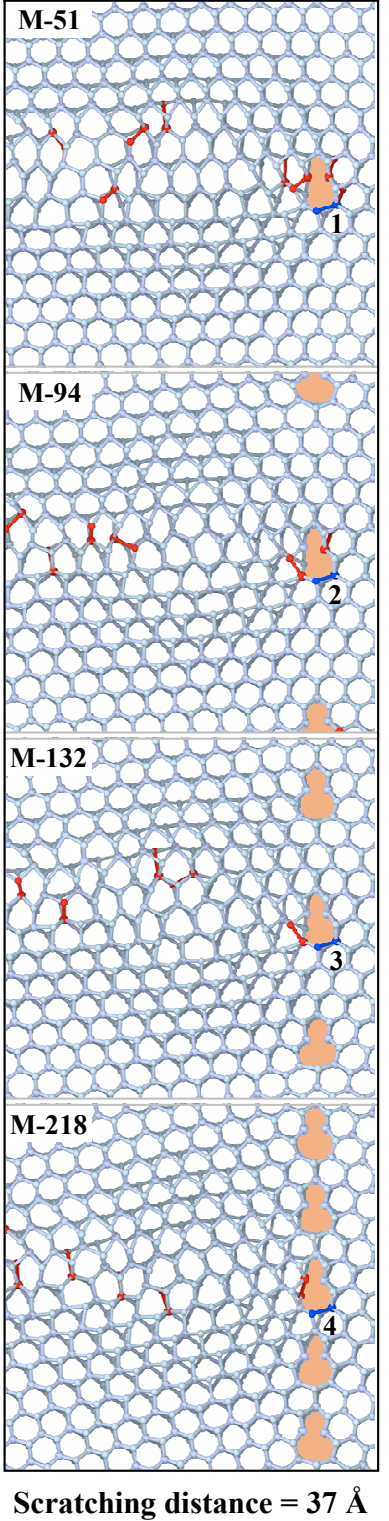

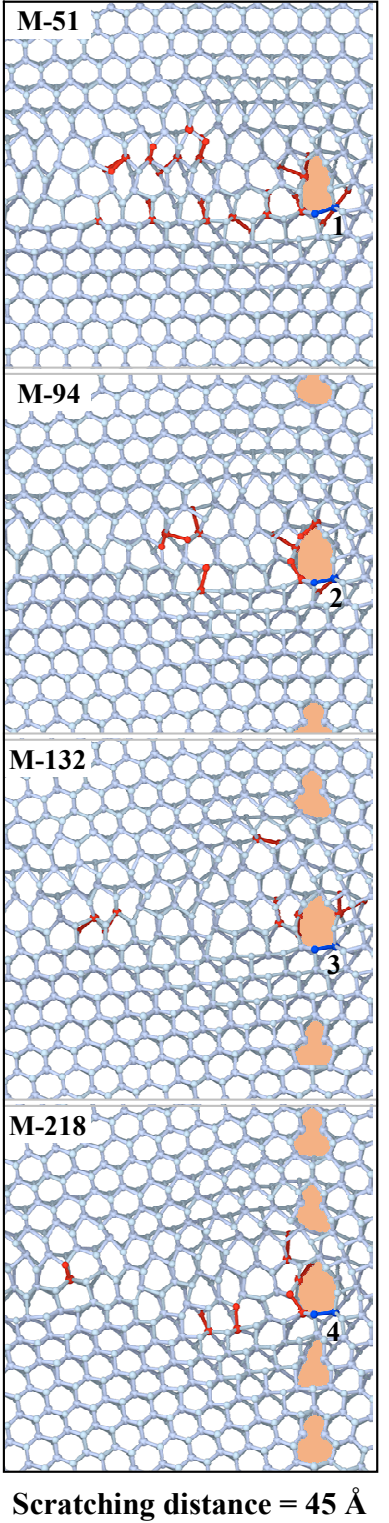

Fig. 7 (a) Relationship between average friction force and the scratching depth when the tip scratches on bare Pt substrate, pristine $\mathrm{MoS}_{2}$ and $\mathrm{MoS}_{2}$ with GB defects. (b) Variation of the average number of long Mo-S bonds with the scratching distance in M-51 model. The number of long bonds is averaged with an interval of $1 \AA$. The diamond tip is positioned at an initial distance of $50 \AA$ from the GB along the $x$ direction. (c) Relationship between the length of the critical bond 1-4 and scratching distance. (d) At the load depth of $5.4 \AA$, the distributions of the long Mo-S bonds for M-51, M-94, M-132 and M-218 models at two sliding distances of 37 and $45 \AA$, respectively. The long Mo-S bonds are marked in red, and the Mo5|7 pairs along the GB defects are colored with bisque. The critical bonds within GBs are numbered by 1-4 and highlighted in blue.

during the scratching process than in uniaxial tensile tests. More complex wear failure mechanisms of GB defects-containing $\mathrm{SL}-\mathrm{MoS}_{2}$ could be investigated, which is concerning on the effect of misorientation angle.

To further explore the wear failure mechanisms, the other Mo5l7- and S5l7-containing models were also scratched under the critical breaking load of M-51 and S-51 models, respectively. The characteristics of $\mathrm{Mo}-\mathrm{S}$ bonds were further considered. Here, the critical bond length of Mo-S bonds is defined as $2.75 \AA$, which is greater than the longest length of the pre-strain Mo-S bond within GB defects-containing SL-MoS2 at equilibrium. 
Table 1 Longest pre-strain Mo-S bond length at equilibrium and the critical breaking load for wear failure.

\begin{tabular}{ccc}
\hline Model & $\begin{array}{c}\text { Longest pre-strain } \\
\text { bond length }(\AA)\end{array}$ & $\begin{array}{c}\text { Critical breaking } \\
\text { load }(\mathrm{nN})\end{array}$ \\
\hline M-51 & 2.73 & 72.77 \\
M-94 & 2.70 & 77.67 \\
M-132 & 2.69 & 82.94 \\
M-218 & 2.67 & 89.40 \\
S-51 & 2.74 & 65.37 \\
S-94 & 2.73 & 74.29 \\
S-132 & 2.72 & 78.09 \\
S-218 & 2.69 & 83.69 \\
Pristine $\mathrm{MoS}_{2}$ & 2.61 & 94.35 \\
\hline
\end{tabular}

In the contact area between the SL-MoS 2 and the tip, the long Mo-S bonds were tracked (bond length is longer than $2.75 \AA$ but less than the maximum cutoff radius of $\mathrm{MoS}_{2}$ ). The distributions of long Mo-S bonds at two scratching distances of 37 and $45 \AA$ were shown in Fig. 7(d). For all Mo5I7-containing models, the long Mo-S bonds were scattered in the contact area at a scratching distance of $37 \AA$. When the tip scratched to $45 \AA$, most of the long Mo-S bonds emerged around the GB of M-51 model. In addition, the quantity of the long bonds around the GB defects decreased with the increase of the misorientation angle. The average number of the long Mo-S bonds was calculated to show the variation trend of the long bonds, as shown in Fig. 7(b). It should be noted that the number of long Mo-S bonds does not include the broken bonds. As the tip scratches to the GB defects, the quantity of long Mo-S bonds increases. But its growth trend decreases with increasing misorientation angle. For low-angle GB defects, the accumulation of those long bonds leads to the permanent break of critical bond-1, as shown in Figs. 7(c) and S7(c) in ESM. But for other models with large-angle GBs, the critical bonds are able to recover after the diamond tip slides over the GBs under the same load, which stops the further damage of $\mathrm{MoS}_{2}$. Therefore, for low-angle GB defects, more Mo-S long bonds translate into the broken bonds when the tip scratches to GB defects, which can confirm that the accumulation of the long bonds around the GB defects leads to the lower wear resistance of GB defects-containing SL-MoS2.

\subsection{Weakening the negative effects of GB defects on the tribological properties of $\mathrm{MoS}_{2}$}

According to the above discussions, the existence of GBs will degrade the tribological properties of $\mathrm{MoS}_{2}$. With the decrease of misorientation angle between $\mathrm{MoS}_{2}$ crystals, the deterioration of GB defects is more pronounced on the tribological properties of $\mathrm{MoS}_{2}$. Therefore, weakening the negative effects of GBs (especially the low-angle GBs) is significant to enhance the quality of $\mathrm{MoS}_{2}$ coatings for their potential applications. Researches show that the adhesive strength of the interfaces could affect the performance of coatings [57, 58]. The adhesive strength of the interfaces between $\mathrm{MoS}_{2}$ and $\mathrm{Pt}$ substrate is changed by altering the LJ parameters, as shown in Tables 2 and S5 in ESM. It should be noted that the parameters of LJ-3 were used in the above simulations. The ideal work of adhesion between $\mathrm{MoS}_{2}$ and Pt substrate could be calculated by the following formula:

$$
W=\frac{E_{1}+E_{2}-E_{12}}{A_{\mathrm{c}}}
$$

where $E_{1}$ and $E_{2}$ are the total energies of the SL-MoS and $\mathrm{Pt}$ substrate at equilibrium, respectively. $E_{12}$ is the total energy of the SL-MoS 2 and Pt substrate when they contact each other at equilibrium. $A_{\mathrm{c}}$ is the contact area of the interface [59].

For the M-51 model, with the interfacial work of adhesion increasing, the critical breaking load for wear reduced as shown in Fig. 8(a). The same effects of the adhesive strength on critical load are observed in S-51 model. From what has been discussed above, the accumulation of those long

Table 2 LJ potential parameters and the work of adhesion.

\begin{tabular}{lccc}
\hline & $\varepsilon_{\mathrm{S}-\mathrm{Pt}}(\mathrm{meV})$ & $\varepsilon_{\mathrm{Mo}-\mathrm{Pt}}(\mathrm{meV})$ & $\begin{array}{c}\text { Work of adhesion } \\
\left(\mathrm{J} / \mathrm{m}^{2}\right)\end{array}$ \\
\hline LJ-1 & 88.92 & 330.71 & 1.03 \\
LJ-2 & 133.38 & 496.06 & 1.57 \\
LJ-3 & 177.84 & 661.41 & 2.13 \\
LJ-4 & 266.76 & 922.12 & 2.64 \\
\hline
\end{tabular}


(a)

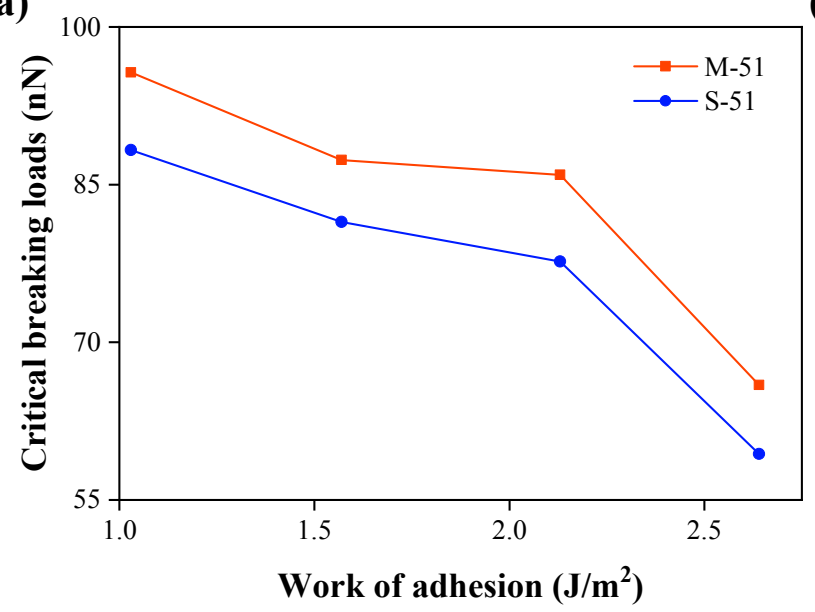

(b)

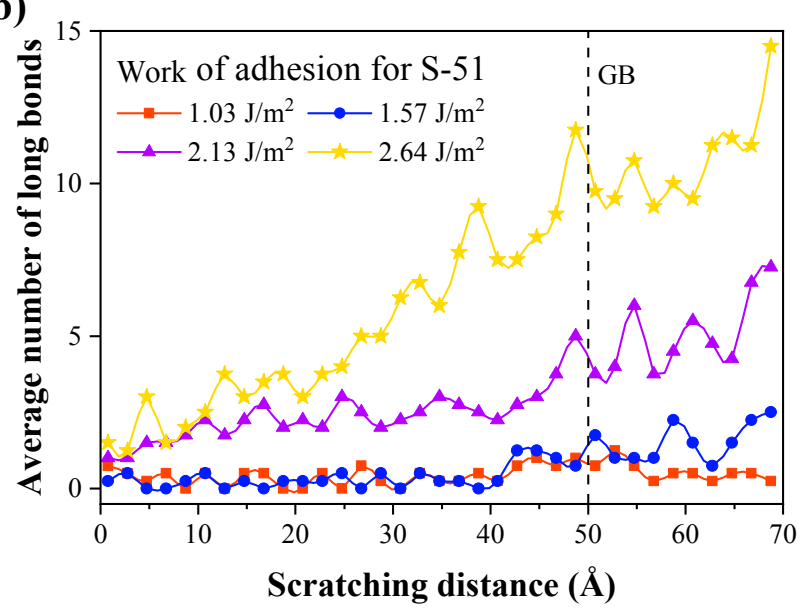

Fig. 8 (a) Critical breaking load for wear failure of M-51 and S-51 model with different work of adhesion between MoS ${ }_{2}$ and Pt substrate. (b) Average number of the long Mo-S bonds of S-51 model with different adhesive strengths at the load depth of $4.6 \AA$.

bonds around the GB defects influences the wear resistance of the $\mathrm{MoS}_{2}$. The average number of long Mo-S bonds under different adhesive strengths has been calculated at same normal load (the critical breaking load of LJ-4), as shown in Fig. 8(b). During the scratching course, the quantity of the long bonds increases with the adhesive strength between $\mathrm{MoS}_{2}$ and Pt substrate. Similar effects of the adhesive strength on the number of the long Mo-S bonds have been observed in M-51 models, as shown in Fig. S8 in ESM. Therefore, a lower adhesion strength of the interface could weaken the negative effects of GB defects on the tribological properties of $\mathrm{MoS}_{2}$. This result is opposite to the previous experiment [60] and simulation works on graphene [45]. They pointed out that the increase of adhesion between substrate and film could suppress the pucker effect $[45,60]$. Although the interface quality could be enhanced by suppressing the pucker effect [61], the resistances of SL-MoS2 and monolayer graphene against the pucker effect are different. The lower out-of-plane bending stiffness of 2D materials is the major causes of the pucker effect. Compared with monolayer graphene, the SL-MoS2 shows higher bending stiffness owing to the larger thickness and the angular and pairwise interactions between Mo and S atoms [9]. The SL-MoS 2 presents an advantage over graphene on resisting against puckering owing to its bending modulus is about 7 times higher than graphene [30]. In addition, Yang et al. [58] observed the controllable friction of SL-MoS 2 on different substrates and found that greater adhesion between substrate and SL-MoS 2 film resulting in higher friction force, which was consistent with the results in this work. Therefore, there could be other major reasons instead of the pucker effect for the increase of wear resistance of GB defects-containing SL-MoS2 by decreasing the adhesion strength.

In order to further explain the effects of the adhesion strength on the wear resistance of GB defects-containing SL-MoS2, the structural deformations of $\mathrm{MoS}_{2}$ have been studied during the scratching process, as shown in Fig. 9. The out-of-plane compressive and in-plane tensile deformation of $\mathrm{MoS}_{2}$ could be described by $L_{\mathrm{Mo}-\mathrm{Mo}}$ and $L_{S-S}$ as shown in Figs. 9(b) and 9(c). It should be noted that for smaller $L_{\mathrm{S}-\mathrm{S}}$, the corresponding out-of-plane compression deformation is larger. With the adhesion strength increasing, the value of $L_{\mathrm{Mo}-\mathrm{Mo}}$ rises to a high level which indicates that the $\mathrm{MoS}_{2}$ is suffering from a larger in-plane tensile deformation. The reduction of $L_{\mathrm{S}-\mathrm{S}}$ is also responsible to more out-of-plane compressive deformation of $\mathrm{MoS}_{2}$. Thus, both the in-plane tensile and out-of-plane compression deformations of $\mathrm{MoS}_{2}$ increase with the adhesion strength, resulting in the rupture of

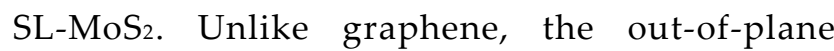
compression deformation of the $\mathrm{SL}-\mathrm{MoS}_{2}$ is the main cause of the rupture Pt-supported SL-MoS 2 
(a)

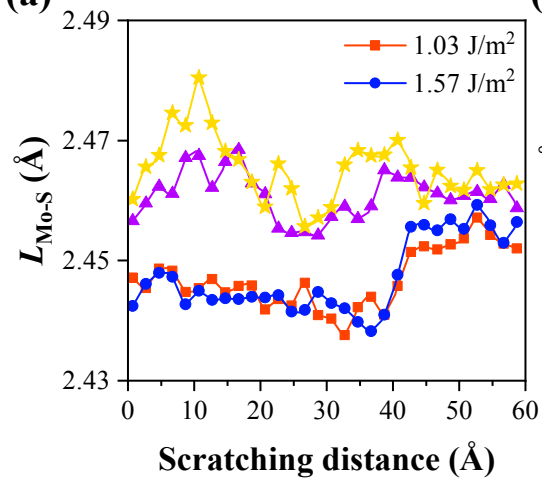

(b)

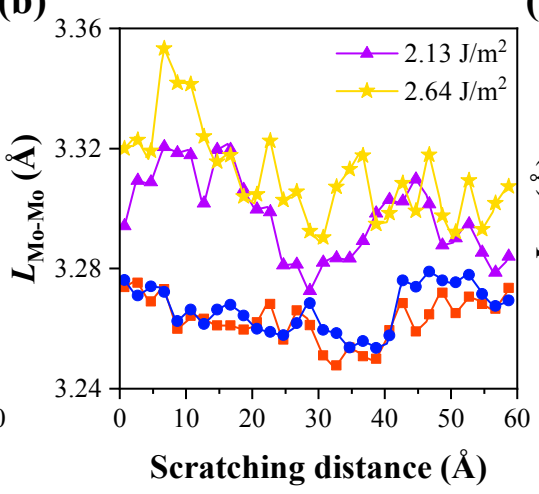

(c)

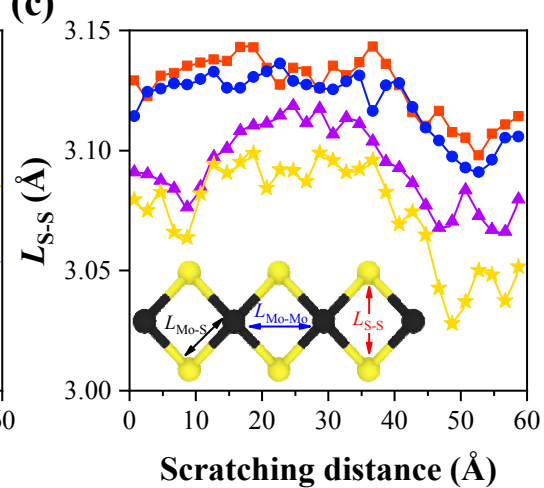

Fig. 9 Relation curves of (a) $L_{\mathrm{Mo}-\mathrm{S}}$, (b) $L_{\mathrm{Mo}-\mathrm{Mo}}$, and (c) $L_{\mathrm{S}-\mathrm{S}}$ with scratching distance in S-51 model with different work of adhesion at a load depth of $4.6 \AA$.

[30, 50]. Therefore, SL-MoS2 undergoes severe structure deformations under a high adhesion strength between SL-MoS 2 and substrate, resulting in more serious wear failure of GB defects-containing SL-MoS2. The above reasons could explain the different findings in this work and previous works on graphene $[45,60]$. That is, the adhesion strength changes the wear resistance of GB defects-containing $\mathrm{SL}_{-}-\mathrm{MoS}_{2}$ by influencing the structure deformations of $\mathrm{MoS}_{2}$. Therefore, the wear resistance of GB defects-containing SL-MoS 2 could be enhanced by decreasing its interfacial adhesion strength to the substrate under certain conditions. A number of efforts have been made to reduce the adhesion strength between $\mathrm{MoS}_{2}$ and substrate, such as the post-processing of vacuum plasma, increasing the layers of $\mathrm{MoS}_{2}$ and deposition of $\mathrm{MoS}_{2}$ on oxides $\left(\mathrm{SiO}_{2}\right.$ or $\left.\mathrm{Al}_{2} \mathrm{O}_{3}\right)$ substrate [58], which can offer a way to improve the wear resistance of $\mathrm{MoS}_{2}$ with low-angle GB defects.

\section{Conclusions}

In this work, the effects of GB defects on the tribological behavior of $\mathrm{MoS}_{2}$ have been investigated by using MD simulations. The GB defects-containing $\mathrm{MoS}_{2}$ is more easily broken during the scratching course owing to the combined effect of pushing and interlocking between the diamond tip and $\mathrm{MoS}_{2}$ atoms. In contrast, GB defects-containing $\mathrm{MoS}_{2}$ is able to withstand a larger load during the indentation process owing to the lack of relative sliding between the interfaces of the tip and $\mathrm{MoS}_{2}$. The wear resistance and carrying capacity of $\mathrm{MoS}_{2}$ with GB defects are relevant to the misorientation angle. Compared with the pristine SL-MoS2, the critical breaking load of GB defects-containing $\mathrm{MoS}_{2}$ with a low misorientation angle is significantly reduced. It should be noted that the critical breaking loads for wear failure on M-51 and S-51 GBs are reduced by $22.9 \%$ and $30.7 \%$, respectively. The critical load of $\mathrm{MoS}_{2}$ with GB defects increases with the misorientation angle. The accumulation of the long Mo-S bonds around the GBs results in the wear failure of GB defects-containing SL-MoS2 during the scratching process. The GBs with low misorientation angles are easier to accumulate long bonds than large-angle GBs, which is severely decreased the wear resistance of $\mathrm{MoS}_{2}$. Weakening the adhesion strength between $\mathrm{MoS}_{2}$ and substrate is an efficient way to eliminate the negative effects of GB defects on the wear resistance of $\mathrm{MoS}_{2}$. The adhesion strength changes the wear resistance of GB defects-containing SL-MoS2 by influencing the structure deformations of $\mathrm{MoS}_{2}$. When the interfacial work of adhesion reduces from 2.13 to $1.03 \mathrm{~J} / \mathrm{m}^{2}$, the wear resistance on M-51 and S-51 GBs are increased by $11.3 \%$ and $13.7 \%$, respectively. These results show the importance of controlling the microstructure, such as the low-angle GB defects, and provide guidelines to enhance the wear resistance of GB defects-containing SL-MoS2. 


\section{Acknowledgements}

The authors acknowledge the support of the National Natural Science Foundation of China (Grant No. 51605026).

\section{Electronic Supplementary Material: Supplementary} material is available in the online version of this article at https://doi.org/10.1007/s40544-020-0459-z.

Open Access This article is licensed under a Creative Commons Attribution 4.0 International License, which permits use, sharing, adaptation, distribution and reproduction in any medium or format, as long as you give appropriate credit to the original author(s) and the source, provide a link to the Creative Commons licence, and indicate if changes were made.

The images or other third party material in this article are included in the article's Creative Commons licence, unless indicated otherwise in a credit line to the material. If material is not included in the article's Creative Commons licence and your intended use is not permitted by statutory regulation or exceeds the permitted use, you will need to obtain permission directly from the copyright holder.

To view a copy of this licence, visit http:// creativecommons.org/licenses/by/4.0/.

\section{References}

[1] Novoselov K S, Mishchenko A, Carvalho A, Castro Neto A H. 2D materials and van der Waals heterostructures. Science 353(6298): aac9439 (2016)

[2] Manzeli S, Ovchinnikov D, Pasquier D, Yazyev O V, Kis A. 2D transition metal dichalcogenides. Nat Rev Mater 2(8): 17033 (2017)

[3] Wang Q H, Kalantar-Zadeh K, Kis A, Coleman J N, Strano M S. Electronics and optoelectronics of twodimensional transition metal dichalcogenides. Nature Nanotech 7(11): 699-712 (2012)

[4] Androulidakis C, Zhang K, Robertson M, Tawfick S. Tailoring the mechanical properties of $2 \mathrm{D}$ materials and heterostructures. 2D Mater 5(3): 032005 (2018)

[5] Zhang S, Ma T B, Erdemir A, Li Q Y. Tribology of two-dimensional materials: From mechanisms to modulating strategies. Mater Today 26: 67-86 (2019)

[6] Bertolazzi S, Brivio J, Kis A. Stretching and breaking of ultrathin $\mathrm{MoS}_{2}$. Acs Nano 5(12): 9703-9709 (2011)

[7] Stewart J A, Spearot D E. Atomistic simulations of nanoindentation on the basal plane of crystalline molybdenum disulfide $\left(\mathrm{MoS}_{2}\right)$. Modelling Simul Mater Sci Eng 21(4): 045003 (2013)

[8] Castellanos-Gomez A, Poot M, Steele G A, van der Zant H S J, Agraït N, Rubio-Bollinger G. Elastic properties of freely suspended $\mathrm{MoS}_{2}$ nanosheets. $A d v$ Mater 24(6): 772-775 (2012)

[9] Jiang J W, Qi Z N, Park H S, Rabczuk T. Elastic bending modulus of single-layer molybdenum disulfide $\left(\mathrm{MoS}_{2}\right)$ : finite thickness effect. Nanotechnology 24(43): 435705 (2013)

[10] Spear J C, Ewers B W, Batteas J D. 2D-nanomaterials for controlling friction and wear at interfaces. Nano Today 10(3): 301-314 (2015)

[11] Lee C, Li Q Y, Kalb W, Liu X Z, Berger H, Carpick R W, Hone J. Frictional characteristics of atomically thin sheets. Science 328(5974): 76-80 (2010)

[12] Chhowalla M, Amaratunga G A J. Thin films of fullerenelike $\mathrm{MoS}_{2}$ nanoparticles with ultra-low friction and wear. Nature 407(6801): 164-167 (2000)

[13] Furlan K P, de Mello J D B, Klein A N. Self-lubricating composites containing $\mathrm{MoS}_{2}$ : A review. Tribol Int 120: 280-298 (2018)

[14] Kong N, Wei B Y, Li D S, Zhuang Y, Sun G P, Wang B. A study on the tribological property of $\mathrm{MoS}_{2} / \mathrm{Ti}-\mathrm{MoS}_{2} /$ $\mathrm{Si}$ multilayer nanocomposite coating deposited by magnetron sputtering. Rsc Adv 10(16): 9633-9642 (2020)

[15] Zeng X, Peng Y, Lang H, Yu K. Probing the difference in friction performance between graphene and $\mathrm{MoS}_{2}$ by manipulating the silver nanowires. $J$ Mater Sci 54(1): 540-551 (2019)

[16] Khac B C, Chung K H. Quantitative assessment of friction characteristics of single-layer $\mathrm{MoS}_{2}$ and graphene using atomic force microscopy. J Nanosci Nanotech 16(5): 4428-4433 (2016)

[17] Li H, Wang J, Gao S, Chen Q, Peng L, Liu K, Wei X. Superlubricity between $\mathrm{MoS}_{2}$ Monolayers. Adv Mater 29(27): 1701474 (2017)

[18] Dietzel D, Brndiar J, Štich I, Schirmeisen A. Limitations of structural superlubricity: Chemical bonds versus contact size. Acs Nano 11(8): $7642-7647$ (2017)

[19] Lee Y H, Zhang X Q, Zhang W J, Chang M T, Lin C T, Chang K D, Yu Y C, Wang J T W, Chang C S, Li L J, et al. Synthesis of large-area $\mathrm{MoS}_{2}$ atomic layers with chemical vapor deposition. Adv Mater 24(17): 2320- 
2325 (2012)

[20] Najmaei S, Liu Z, Zhou W, Zou X L, Shi G, Lei S D, Yakobson B I, Idrobo J C, Ajayan P M, Lou J. Vapour phase growth and grain boundary structure of molybdenum disulphide atomic layers. Nat Mater 12(8): 754-759 (2013)

[21] Yu Z G, Zhang Y W, Yakobson B I. An anomalous formation pathway for dislocation-sulfur vacancy complexes in polycrystalline monolayer $\mathrm{MoS}_{2}$. Nano Lett 15(10): 6855-6861 (2015)

[22] Lu C P, Li G, Mao J, Wang L M, Andrei E Y. Bandgap, mid-gap states, and gating effects in $\mathrm{MoS}_{2}$. Nano Lett 14(8): 4628-4633 (2014)

[23] Lin Z, Carvalho B R, Kahn E, Lv R T, Rao R, Terrones $\mathrm{H}$, Pimenta M A, Terrones M. Defect engineering of two-dimensional transition metal dichalcogenides. $2 D$ Mater 3(2): 022002 (2016)

[24] Yazyev O V, Chen Y P. Polycrystalline graphene and other two-dimensional materials. Nature Nanotech 9(10): 755-767 (2014)

[25] Chen S, Gao J F, Srinivasan B M, Zhang G, Yang M, Cha J W, Wang S J, Chi D Z, Zhang Y W. Revealing the grain boundary formation mechanism and kinetics during polycrystalline $\mathrm{MoS}_{2}$ growth. Acs Appl Mater Interfaces 11(49): 46090-46100 (2019)

[26] Liu Z, Amani M, Najmaei S, Xu Q, Zou X L, Zhou W, Yu T, Qiu C Y, Birdwell A G, Crowne F J, et al. Strain and structure heterogeneity in $\mathrm{MoS}_{2}$ atomic layers grown by chemical vapour deposition. Nat Commun 5: 5246 (2014)

[27] Zhou W, Zou X L, Najmaei S, Liu Z, Shi Y M, Kong J, Lou J, Ajayan P M, Yakobson B I, Idrobo J C. Intrinsic structural defects in monolayer molybdenum disulfide. Nano Lett 13(6): 2615-2622 (2013)

[28] van der Zande A M, Huang P Y, Chenet D A, Berkelbach T C, You Y M, Lee G H, Heinz T F, Reichman D R, Muller D A, Hone J C. Grains and grain boundaries in highly crystalline monolayer molybdenum disulphide. Nat Mater 12(6): 554-561 (2013)

[29] Huang Y H, Yao Q Z, Lu Z X, Jiao L Y, Zhang S, Li Q $\mathrm{Y}$, Meng Y G. Antiwear performance of monolayer $\mathrm{MoS}_{2}$ modulated by residual straining. Acs Appl Nano Mater 1(12): 7092-7097 (2018)

[30] Kong N, Wei B Y, Zhuang Y, Zhang J, Li H B, Wang B. Effect of compressive prestrain on the anti-pressure and anti-wear performance of monolayer $\mathrm{MoS}_{2}$ : a molecular dynamics study. Nanomaterials 10(2): 275 (2020)

[31] Wang C Q, Li H S, Zhang Y S, Sun Q, Jia Y. Effect of strain on atomic-scale friction in layered $\mathrm{MoS}_{2}$. Tribol Int 77: 211-217 (2014)

[32] Cao X A, Gan X H, Lang H J, Yu K, Ding S Y, Peng Y
T, Yi W M. Anisotropic nanofriction on $\mathrm{MoS}_{2}$ with different thicknesses. Tribol Int 134: 308-316 (2019)

[33] Onodera T, Morita Y, Nagumo R, Miura R, Suzuki A, Tsuboi H, Hatakeyama N, Endou A, Takaba H, Dassenoy F, et al. A computational chemistry study on friction of h-MoS 2 Part II friction anisotropy. $J$ Phys Chem B 114(48): 15832-15838 (2009)

[34] Sheehan P E, Lieber C M. Friction between van der Waals solids during lattice directed sliding. Nano Lett 17(7): 4116-4121 (2017)

[35] Zhao X Y, Phillpot S R, Sawyer W G, Sinnott S B, Perry S S. Transition from thermal to athermal friction under cryogenic conditions. Phys Rev Lett 102(18): 186102 (2009)

[36] Dang K Q, Spearot D E. Effect of point and grain boundary defects on the mechanical behavior of monolayer $\mathrm{MoS}_{2}$ under tension via atomistic simulations. $J$ Appl Phys 116(1): 013508 (2014)

[37] Wu J Y, Cao P Q, Zhang Z S, Ning F L, Zheng S S, He J Y, Zhang Z L. Grain-size-controlled mechanical properties of polycrystalline monolayer $\mathrm{MoS}_{2}$. Nano Lett 18(2): 1543-1552 (2018)

[38] Li M, Wan Y, Tu L, Yang Y, Lou J. The effect of $\mathrm{VMoS}_{3}$ point defect on the elastic properties of monolayer $\mathrm{MoS}_{2}$ with REBO potentials. Nanoscale Res Lett 11(1): 155 (2016)

[39] Ky D L C, Tran Khac B C, Le C T, Kim Y S, Chung K H. Friction characteristics of mechanically exfoliated and CVD-grown single-layer $\mathrm{MoS}_{2}$. Friction 6(4): 395-406 (2018)

[40] Lavini F, Calò A, Gao Y, Albisetti E, Li T D, Cao T, Li G, Cao L, Aruta C, Riedo E. Friction and work function oscillatory behavior for an even and odd number of layers in polycrystalline $\mathrm{MoS}_{2}$. Nanoscale 10(17): 8304-8312 (2018)

[41] Zou X L, Liu Y Y, Yakobson B I. Predicting dislocations and grain boundaries in two-dimensional metal-disulfides from the first principles. Nano Lett 13(1): 253-258 (2013)

[42] Dong Y, Li Q, Martini A. Molecular dynamics simulation of atomic friction: A review and guide. J Vac Sci Technol A 31(3): 033108 (2013)

[43] Sinnott S, Heo S J, Brenner D, Harrison J. Computer Simulations of Nanometer-Scale Indentation and Friction. In Nanotribology and Nanomechanics. Springer Berlin Heidelberg, 2007: 1051-1106.

[44] Sun X L, Wang Z G, Fu Y Q. Defect-mediated lithium adsorption and diffusion on monolayer molybdenum disulfide. Sci Rep-UK 5: 18712 (2015)

[45] Zhang J, Chen X, Xu Q, Ma T, Hu Y, Wang H, Tieu A $\mathrm{K}$, Luo J. Effects of grain boundary on wear of 
graphene at the nanoscale: A molecular dynamics study. Carbon 143: 578-586 (2019)

[46] Carlsson J M, Ghiringhelli L M, Fasolino A. Theory and hierarchical calculations of the structure and energetics of [0001] tilt grain boundaries in graphene. Phys Rev B 84(16): 165423 (2011)

[47] Brenner D W, Shenderova O A, Harrison J A, Stuart S J, $\mathrm{Ni}$ B, Sinnott S B. A second-generation reactive empirical bond order (REBO) potential energy expression for hydrocarbons. J Phys-Condens Mat 14(4): 783-802 (2002)

[48] Liang T, Phillpot S R, Sinnott S B. Parametrization of a reactive many-body potential for Mo-S systems. Phys Rev B 79(24): 245110 (2009)

[49] Xiong S, Cao G X. Molecular dynamics simulations of mechanical properties of monolayer $\mathrm{MoS}_{2}$. Nanotechnology 26(18): 185705 (2015)

[50] Liu Y, Liu Y H, Ma T B, Luo J B. Atomic scale simulation on the anti-pressure and friction reduction mechanisms of $\mathrm{MoS}_{2}$ monolayer. Materials 11(5): 683 (2018)

[51] Wang L, Duan F. Nanoscale wear mechanisms of fewlayer graphene sheets induced by interfacial adhesion. Tribol Int 123: 266-272 (2018)

[52] Xu Q, Li X, Zhang J, Hu Y, Wang H, Ma T. Suppressing Nanoscale Wear by Graphene/Graphene Interfacial Contact Architecture: A Molecular Dynamics Study. Acs Appl Mater Inter 9(46): 40959-40968 (2017)

[53] Sandoz-Rosado E J, Tertuliano O A, Terrell E J. An atomistic study of the abrasive wear and failure of

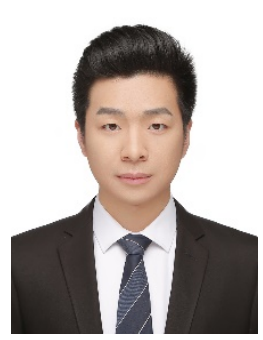

Boyu WEI. He received his bachelor degree in 2018 in Beijing Technology and Business University, China. Then, he is a master graduate student in the School of Mechanical Engineering, University of Science and Technology

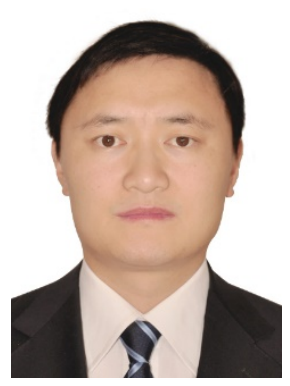

Ning KONG. He received his Ph.D. degree in mechanical engineering from University of Wollongong, Australia in 2015. He joined the University of Science and Technology Beijing from 2015. His current position is an associate professor. His graphene sheets when used as a solid lubricant and a comparison to diamond-like-carbon coatings. Carbon 50(11): 4078-4084 (2012)

[54] Pena-Alvarez M, del Corro E, Morales-Garcia A, Kavan L, Kalbac M, Frank O. Single Layer Molybdenum Disulfide under Direct Out-of-Plane Compression: Low-Stress Band-Gap Engineering. Nano Lett 15(5): 3139-3146 (2015)

[55] Mo Y F, Turner K T, Szlufarska I. Friction laws at the nanoscale. Nature 457(7233): 1116-1119 (2009)

[56] Liu T H, Gajewski G, Pao C W, Chang C C. Structure, energy, and structural transformations of graphene grain boundaries from atomistic simulations. Carbon 49(7): 2306-2317 (2011)

[57] Zeng X Z, Peng Y T, Lang H J. A novel approach to decrease friction of graphene. Carbon 118: 233-240 (2017)

[58] Yang J J, Liu L. Nanotribological properties of 2-D $\mathrm{MoS}_{2}$ on different substrates made by atomic layer deposition (ALD). Appl Surf Sci 502: 144402 (2020)

[59] Kisin S, Vukic J B, van der Varst P G T, de With G, Koning C E. Estimating the polymer-metal work of adhesion from molecular dynamics simulations. Chem Mater 19(4): 903-907 (2007)

[60] Zhao S J, Zhang Z H, Wu Z H, Liu K H, Zheng Q S, Ma M. The Impacts of Adhesion on the Wear Property of Graphene. Adv Mater Interfaces 6(18): 1900721(2019)

[61] Li S Z, Li Q Y, Carpick R W, Gumbsch P, Liu X Z, Ding X D, Sun J, Li J. The evolving quality of frictional contact with graphene. Nature 539(7630): 541-545 (2016)

Beijing, China. His interests include friction and wear behavior of 2D materials and molecular dynamics simulation.

research areas cover tribology (friction, lubrication, and wear), hot rolling technology, materials engineering, computational mechanics (FEM and MD), nanotechnology, and structural design and analysis with various backgrounds. 